\title{
Low-rank Factorization of Electron Integral Tensors and Its Application in Electronic Structure Theory
}

\author{
Bo Peng*, Karol Kowalski**
}

William R. Wiley Environmental Molecular Sciences Laboratory, Battelle, Pacific Northwest National Laboratory, K8-91, P.O. Box 999, Richland, WA 99352, USA

\begin{abstract}
In this letter, we apply reverse Cuthill-McKee (RCM) algorithm to transform two-electron integral tensors to their block diagonal forms. By further applying Cholesky decomposition (CD) on each of the diagonal blocks, we are able to represent the high-dimensional two-electron integral tensors in terms of permutation matrices and low-rank Cholesky vectors. This representation facilitates low-rank factorizations of high-dimensional tensor contractions in postHartree-Fock calculations. Here, we discuss the second-order Møller-Plesset (MP2) method and the linear coupled-cluster model with doubles (L-CCD) as examples to demonstrate the efficiency of this technique in representing the two-electron integrals in a compact form.

Keywords: electronic structure theory, two-electron integral tensor, tensor contraction, low-rank factorization, reverse Cuthill-McKee, Cholesky decomposition
\end{abstract}

\section{Introduction}

Expensive tensor contractions are often the bottleneck of the accurate electronic structure calculations. A typical example is the coupled-cluster (CC)

\footnotetext{
* Corresponding author

${ }^{* *}$ Corresponding author

Email addresses: peng398@pnnl.gov (Bo Peng), karol.kowalski@pnnl.gov (Karol Kowalski)
}

Preprint submitted to Chem. Phys. Lett.

January 18, 2017

(C) 2017. This manuscript version is made available under the Elsevier user license http://www.elsevier.com/open-access/userlicense/1.0/ 
model.[1, 2, 3, 4, 5, 6, 7] Even though its accuracy can be systematically im5 proved by including higher excitations in an iterative or perturbative manner, high numerical overhead and high memory requirements associated with performing contractions between high dimensionality tensors representing cluster amplitudes and two-electron integrals tensors in a parallel implementation precludes this formalism from being applied to large systems. For example, the $\operatorname{CCSD}(\mathrm{T})$ approach[8] scales as $\mathcal{O}\left(N^{7}\right)$ with $N$ representing the system size, and the corresponding memory requirements are proportional to $N^{4}$. To reduce the high numerical overhead and high memory demands of the CC methods, low-rank representations of the involved tensors are highly expected. Since the amplitudes tensors defining the corresponding wave function are only known

15 after achieving convergence in the iterative process, the a-priori decomposition of the cluster amplitudes, given highly non-linear character of the CC equations, may be a challenging task. Instead, in this letter we will focus on low-rank representations of the two-electron integral tensors which can be calculated prior to the CC calculations. Typically, this can be done by invoking well-established procedures such as the density-fitting $[9,10,11,12,13,14,15,16]$ or incomplete pivoted Cholesky decomposition (CD) $[17,18,19,20]$ methods to represent the atomic two-electron integral tensor in terms of the products of low-rank tensors. In practice, both methods have their merits, and the CD method may be preferred since the accuracy of the decomposition can be controlled to arbitrary precision. However, the number of the Cholesky vectors resulting from the above approach is usually very large and the cost of the tensor contractions using Cholesky vectors still can lead a considerable numerical effort. Unfortunately, the situation may become even worse when using the $\mathrm{CD}$ technique to decompose a non-diagonally dominant integral tensor (e.g. exchange-type). In this case, the number of the Cholesky vectors can be significantly larger than the size of the basis set.

Remarkably, the two-electron integral tensor will become sparse as system size increases. For a sparse tensor, it is well-known that an optimal reordering of columns/rows can reduce tensor decomposition procedure, thereby reducing 
the space and time required to perform the factorization.[21, 22] Moreover, in the corresponding parallel implementations, the reordering can even improve the parallel performance.[23, 24] In practice, computing the optimal reordering is $N P$-complete, thus heuristic reordering algorithms are often used, such as the minimum degree, the Cuthill-McKee (CM), the reverse Cuthill-McKee (RCM),

40 the Sloan, and the nested dissection orderings. [25, 22, 26, 27, 28] It seems that the potential applications of these algorithms in the areas of electronic structure theory are still under development. To the best of our knowledge, there were only few attempts in the past to apply some of these algorithms in the electronic structure calculations. For example, the RCM algorithm can be sim45 ply applied to reorder the coordinates of the molecular structure to speed up the sparse algebra involved in the Hartree-Fock (HF), density-functional theory (DFT) and second-order perturbation theory (MP2) calculations.[29, 30] Also, in early formulations of density matrix renormalization group (DMRG) formalism for molecular systems, the RCM algorithm was adapted to reorder molecular orbitals. Such reordering (may not be optimal) can significantly affect the convergence and accuracy of the calculations. [31, 32] In this paper, we will demonstrate that by applying the RCM algorithm to the original two-electron integral tensor, an optimal permutation can be found to transform the original tensor to its bandwidth reduced form. It is quite often that this bandwidth ${ }_{55}$ reduced form is actually a block-diagonal form. Then the incomplete pivoted CD can be applied to obtain the low-rank Cholesky vectors that can further be utilized to obtain a low-rank factorization of the tensor contractions in the post-Hartree-Fock calculations. In preliminary tests, the RCM-CD procedure is applied to two post-Hartree-Fock methods: the second order Møller-Plesset theory (MP2) and the linear version of the CC formalism with doubles (L-CCD). 

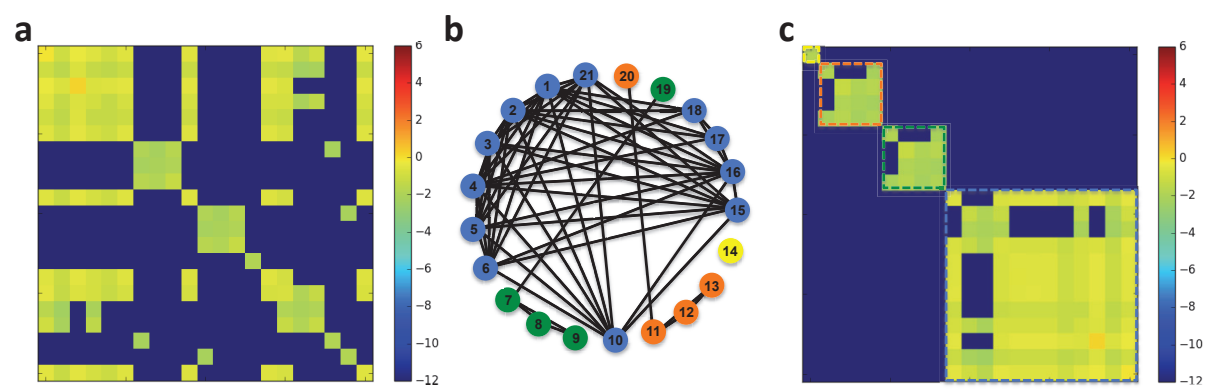

Figure 1: Schematic representation of a two-electron integral tensor (Coulomb-type atomic two-electron integrals for $\mathrm{N}_{2}$ system in STO-3G basis set) transformed from its original form (a) to a block-diagonal form (c) by a permutation generated by analyzing the connectivity of its corresponding graph (b). In (a) and (c), the integral tensor has been mapped onto a twodimensional matrix, whose elements are represented by pixels. All the pixels are highlighted by different colors according to the $\log _{10}$ values of their absolute magnitudes (see the color bar). In (b), the threshold for determining connectivity between any two nodes is $1.0 \times$ $1.0^{-10}$. According to the connectivity in (b), the nodes can be classified into different regimes that show no connectivity with each other. Each regime here is represented by a color and corresponds to a diagonal block (dashed frame) in (c).

\section{Methodology}

The two-electron Coulomb-type integral tensor $(\mathbf{J})$ expressed in terms of atomic orbitals $(\mathrm{AO})$, is denoted as

$$
J_{\mu \nu \lambda \sigma}=(\mu \nu \mid \lambda \sigma)=\iint \phi_{\mu}\left(\mathbf{r}_{1}\right) \phi_{\nu}\left(\mathbf{r}_{1}\right) \frac{1}{\left\|\mathbf{r}_{1}-\mathbf{r}_{2}\right\|} \phi_{\lambda}\left(\mathbf{r}_{2}\right) \phi_{\sigma}\left(\mathbf{r}_{2}\right) \mathrm{d} \mathbf{r}_{1} \mathrm{~d} \mathbf{r}_{2},
$$

where the $\phi$ 's are the $\mathrm{AO}$ basis functions, the subscripts $\mu, \nu, \lambda, \sigma$ are the $\mathrm{AO}$ basis function indices, and $\mathbf{r}_{1}$ and $\mathbf{r}_{2}$ are the Cartesian coordinates of electrons 1 and 2 , respectively. Its exchange-type counterpart $(\mathbf{K})$ is denoted as

$$
K_{\mu \lambda \nu \sigma}=\langle\mu \lambda \mid \nu \sigma\rangle=J_{\mu \nu \lambda \sigma}
$$

Note that both $\mathbf{J}$ and $\mathbf{K}$ are symmetric with respect to basis function pairs, and can be considered as the Gram matrices that are defined by the $L^{2}$ inner product of basis function pairs in the Coulomb metric, $\left\langle\cdot, \frac{1}{\left\|\mathbf{r}_{1}-\mathbf{r}_{2}\right\|}\right\rangle$, which guarantees 65 their positive semidefiniteness. 
In the following discussion, we will adapt the graph representation of matrix to represent the two-electron integral tensors. In the typical graph representation of a matrix, the nodes denote the row/column indices of the matrix, and if the absolute magnitude of a matrix element is larger than a predefined threshold, then the corresponding two nodes are connected by an edge. Now, take $\mathbf{J}$ for an example (similar for $\mathbf{K}$ ), and consider its graph representation (see Fig. 1). We can define each unique basis function pair as a node, and map $\mathbf{J}$ onto a two-dimensional graph (or matrix) for all the nodes with dimension $d=\frac{N(N+1)}{2}$ where $N$ is the number of the AO basis functions. The magnitude of each element in the graph then gives the connectivity information between the two corresponding nodes (Fig. 1b). In order to get the connectivity information of the entire graph, every single element of the AO-based two-electron integral tensor needs to be evaluated, which takes $N^{4}$ operations. In practice, since the evaluation is already done in the prescreening steps during the generation of the AO-based two-electron integral tensor, the construction of the connectivity graph will not bring more operation cost to a typical post-Hartree-Fock calculation. Then, by performing a reordering algorithm on the graph, we can obtain a permutation matrix that transforms the graph to its quasi-diagonal form (Fig. 1c). Here, we employ the well-known reverse Cuthill-McKee algorithm for the reordering. The RCM procedure can be briefly described as follows.

1. Find a starting node, and label it as $\eta_{0}$.

2. For $i=0,1, \cdots, N^{2}-1$, do

(a) Find all the unlabelled neighbors of $\eta_{i}$.

(b) Label these neighbors (start from $\eta_{i+1}$ ) in increasing order of the 90 corresponding degree.

3. The RCM ordering is given by $\left\{\eta_{N^{2}-1}, \eta_{N^{2}-2}, \cdots, \eta_{0}\right\}$.

As discussed and proved in Ref.[33], the complexity of the RCM algorithm depends on the way the sorting (step $2 b$ ) is done. By applying a modified linear-sorting algorithm, the complexity of the RCM algorithm can be reduced to $\mathcal{O}\left(N_{s}\right)$ with $N_{s}$ the number of the elements that are significantly larger than 
the pre-defined prescreening threshold. The $N_{s}$ will be approaching to $\mathcal{O}(N)$ as the system becomes larger. Therefore, the complexity of constructing the connectivity graph and performing the RCM procedure is actually small, especially in comparison with the complexity of the tensor contractions in those post-Hartree-Fock calculations. Additionally, the numerical cost of the RCM procedure can be further reduced by employing the Schwartz-based screening procedures.

In general, since the number of the significant elements in the AO-based twoelectron integral tensor increases linearly with the system size, as the system becomes larger, the AO-based two-electron integral tensor will become sparser, and it will be more likely that the integral tensor corresponds to a disconnected graph given the same threshold for determining the connectivity between any two nodes. In the present study, the threshold is set to $1.0 \times 10^{-10}$ for all the test cases. In the general case (graph is not connected), matrix $\mathbf{J}$ can be put in a quasi-diagonal block form

$$
\mathbf{J}=\mathbf{P}_{\mathbf{J}} \mathbf{B}_{\mathbf{J}} \mathbf{P}_{\mathbf{J}}^{T}
$$

where $\mathbf{P}_{\mathbf{J}}$ is a permutation tensor and $\mathbf{B}_{\mathbf{J}}$ is a block diagonal tensor with $n$ diagonal blocks

$$
\mathbf{B}_{\mathbf{J}}=\left[\begin{array}{cccc}
\mathbf{B}_{\mathbf{J}, 1} & 0 & \cdots & 0 \\
0 & \mathbf{B}_{\mathbf{J}, 2} & \cdots & 0 \\
\vdots & \vdots & \ddots & \vdots \\
0 & 0 & \cdots & \mathbf{B}_{\mathbf{J}, n}
\end{array}\right]=\bigoplus_{k=1}^{n} \mathbf{B}_{\mathbf{J}, k}
$$

and the dimension of the block $\mathbf{B}_{\mathbf{J}, k}$ is $d_{k}$ with $\sum_{k=1}^{n} d_{k}=d$. Since $\mathbf{J}$ is postive semidefinite, so are $\mathbf{B}_{\mathbf{J}}$ and $\mathbf{B}_{\mathbf{J}, i}$ 's. Therefore, an incomplete fully pivoted CD of $\mathbf{J}$,

$$
\mathbf{J}=\sum_{m}^{N_{m}} \mathbf{L}_{\mathbf{J}}^{m}\left(\mathbf{L}_{\mathbf{J}}^{m}\right)^{T},
$$

can be instead done by performing the incomplete pivoted $\mathrm{CD}$ on each diagonal 
block, i.e.

$$
\mathbf{J}=\mathbf{P}_{\mathbf{J}} \bigoplus_{k=1}^{n}\left(\sum_{m}^{N_{k}} \mathbf{L}_{\mathbf{J}, k}^{m}\left(\mathbf{L}_{\mathbf{J}, k}^{m}\right)^{T}\right) \mathbf{P}_{\mathbf{J}}^{T}
$$

with each block decomposed as $\mathbf{B}_{\mathbf{J}, k}=\sum_{m}^{N_{k}} \mathbf{L}_{\mathbf{J}, k}^{m}\left(\mathbf{L}_{\mathbf{J}, k}^{m}\right)^{T}$ and the number of the Cholesky vectors $N_{m}=\sum_{k=1}^{n} N_{k}$. Note that the cost of Eq. (5) is usually $\mathcal{O}\left(N_{m} d^{2}\right)$, which in the RCM algorithm (i.e. if Eq. (6) is performed) will be reduced to $\mathcal{O}\left(\sum_{k=1}^{n} N_{k} d_{k}^{2}\right)$. More importantly, the Cholesky matrix of $\mathbf{J}, \mathbf{L}_{\mathbf{J}}$, now can be formatted in a blocking manner

$$
\mathbf{L}_{\mathbf{J}}=\mathbf{P}_{\mathbf{J}} \mathbf{L}_{\mathbf{B}} \quad \text { and } \quad \mathbf{L}_{\mathbf{B}}=\bigoplus_{k=1}^{n} \mathbf{L}_{\mathbf{J}, k}
$$

with $\mathbf{L}_{\mathbf{J}, \mathbf{B}}$ the block Cholesky matrix and $\mathbf{L}_{\mathbf{J}, k}$ the Cholesky matrix of the diagonal block $k$.

\section{Applications}

In this section, we will choose the MP2 and the L-CCD methods as two examples to show how this factorization facilitates the low-rank factorization of the tensor contractions in the electronic structure calculations. The ideas can be easily extended to more accurate and sophisticated post-Hartree-Fock methodologies including ground- and excited-state formulations. All the calculations have been performed using NWChem suite of codes.[34]

In the MP2 calculation, the time limiting step is the integral tensor transformation from the $\mathrm{AO}$ space to the molecular orbital (MO) space,

$$
\tilde{J}_{i a j b}=(i a \mid j b)=\sum_{\mu, \nu, \lambda, \sigma}^{N} C_{\mu, i} C_{\nu, a} C_{\lambda, j} C_{\sigma, b} J_{\mu \nu \lambda \sigma},
$$

where $\mathbf{C}$ is the $\mathrm{MO}$ coefficient matrix, and the subscripts $i, j, \cdots$ and $a, b, \cdots$ representing occupied and virtual MOs, respectively. Usually this step is carried out in four steps where each step is characterized by $\mathcal{O}\left(N^{5}\right)$ scaling. By applying the Eqs. (6) and (7) to the Eq. (8), and after some derivations we have

$$
\tilde{J}_{i a j b}=\sum_{k}^{n} \sum_{m}^{N_{k}} X_{k}(i, a, m) X_{k}(j, b, m)
$$




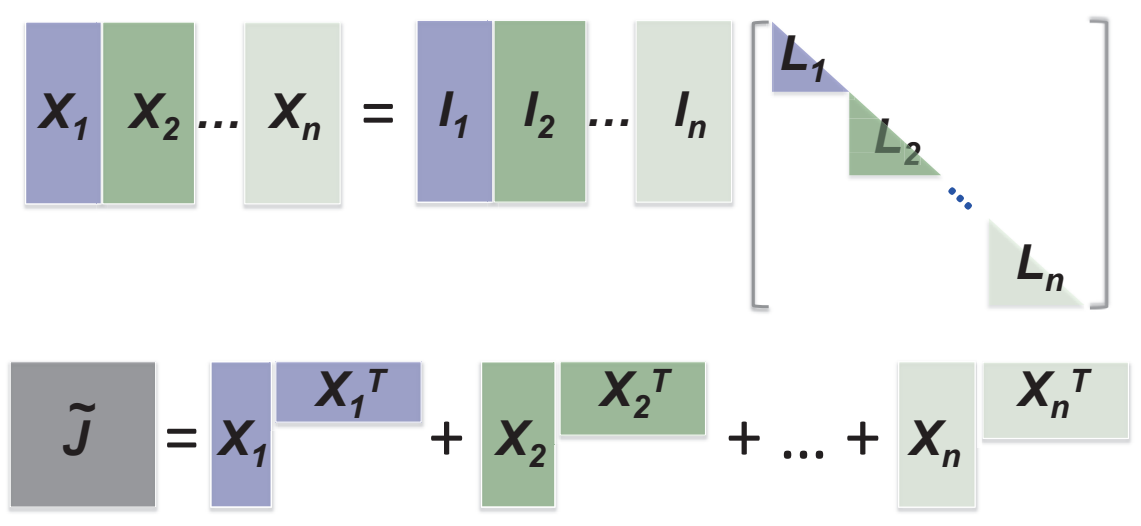

Figure 2: Block representation of the two-electron integral tensor (Coulomb-type) transformation from the AO space to the MO space. $\tilde{\mathbf{J}}$ is the MO-based integral tensor. $\mathbf{X}$ and $\mathbf{I}$ are intermediate blocks. L's are block-wise Cholesky vectors.

with intermediates $\mathbf{X}$ and $\mathbf{I}$ defined as

$$
\begin{aligned}
X_{k}(i, a, m) & =\sum_{x}^{d_{k}} I(i, a, x) L_{J, k}(x, m), \\
I(i, a, x) & =\sum_{\mu, \nu}^{N} C_{\mu, i} C_{\nu, a} P_{J}(\mathcal{P}, x),
\end{aligned}
$$

with super-index $\mathcal{P} \equiv(\mu, \nu)$ and $x$ representing the components of the Cholesky vectors of the $\mathbf{B}_{\mathbf{J}, k}$ matrix. The costs of Eqs. (9), (10), and (11) are $\mathcal{O}\left(N_{m} N_{o}^{2} N_{v}^{2}\right)$, $\mathcal{O}\left(N_{o} N_{v} N_{k} d_{k}\right)$, and $\mathcal{O}\left(N_{o}^{2} d_{k}\right)$ respectively. Note that the existence of the permutation matrix can make the calculation of the intermediate $I(i, a, x)$ really fast. More importantly, the Eqs. (9), (10), and (11) give the low-rank working equations of the integral transformation, and can be made block-wise (see Fig. 2). The Eqs. (9), (10), and (11) can also be applied in other post-Hartree-Fock calculations. For example, the tensor $\tilde{J}_{i a j b}$ will also be used in the CC calculations. In this case, the time limiting step becomes different contractions between the two-electron integral tensor and the double amplitude tensor, rather than the AO-to-MO transformation. Thus, the Eq. (9) can then be alternatively done in the MO space (the intermediate $\mathbf{I}$ then becomes a permutation matrix). Usually, as can be seen in the later discussion, the rank of the Cholesky vectors 
obtained by this way (i.e. the decomposition in MO space) will be smaller.

For L-CCD, the most time-consuming term is

$$
\sum_{e, f}^{N_{v}} \tilde{K}_{a b e f} t_{i j}^{e f}=\sum_{e, f}^{N_{v}}\langle a b \mid e f\rangle t_{i j}^{e f}
$$

which scales as $\mathcal{O}\left(N_{o}^{2} N_{v}^{4}\right)$. Now, similar to the Coulomb-type two-electron integral $\mathbf{J}$ (Eqs. (3) and (4)), a permutation $\mathbf{P}_{\mathbf{K}}$ that is found by the RCM procedure can be applied to the exchange-type two-electron integral $(\mathbf{K})$ to obtain a quasi-diagonal form $\mathbf{B}_{\mathbf{K}}$. If this quasi-diagonal form is block-diagonal, we can then apply the incomplete pivoted CD on each block, and the obtained Cholesky vector $\mathbf{L}_{\mathbf{K}}$ will have a similar form to Eq. (7). Accordingly, we can reformulate the contraction term as

$$
\sum_{e, f}^{N_{v}}\langle a b \mid e f\rangle t_{i j}^{e f}=\sum_{k}^{n} \sum_{m}^{N_{k}} U_{k}(a, b, m) V_{k}(i, j, m),
$$

where the intermediates $\mathbf{U}$ and $\mathbf{V}$ are defined as

$$
\begin{aligned}
& U_{k}(a, b, m)=\sum_{x}^{d_{k}} I^{\prime}(a, b, x) L_{K, k}(x, m), \\
& V_{k}(i, j, m)=\sum_{x}^{d_{k}} L_{K, k}(x, m) I^{\prime \prime}(i, j, x),
\end{aligned}
$$

with

$$
\begin{aligned}
& I^{\prime}(a, b, x)=\sum_{\mu, \nu}^{N} C_{\mu, a} C_{\nu, b} P_{K}(\mathcal{P}, x), \\
& I^{\prime \prime}(i, j, x)=\sum_{e, f}^{N_{v}} I^{\prime}(e, f, x) t_{i j}^{e f} .
\end{aligned}
$$

The costs of Eqs. (13)-(17) are $\mathcal{O}\left(N_{m} N_{o}^{2} N_{v}^{2}\right), \mathcal{O}\left(N_{v} N_{v} N_{k} d_{k}\right), \mathcal{O}\left(N_{o} N_{o} N_{k} d_{k}\right)$, $\mathcal{O}\left(N_{v}^{2} d_{k}\right)$, and $\mathcal{O}\left(N_{o}^{2} N_{v}^{2} d_{k}\right)$, respectively. Similar to the Eqs. (9), (10), and (11), the Eqs. (12)-(17) can also be made block-wise. Note that since the exchangetype integral tensor is not diagonal dominant, the direct $\mathrm{CD}$ will not provide any computational savings, and the rank of the obtained Cholesky vector, if done, can be as large as $N^{2}$. Actually, the rank can be at least reduced to $N_{v}^{2}$ if 
Table 1: Dimension of each block in the block-diagonal AO-based integral tensor generated for $\mathrm{N}_{2}$ molecule with various basis sets. The number of the Cholesky vectors are given in the parenthesis. The truncation threshold in incomplete pivoted CD is set to $1.0 \times 10^{-10} . d$ is the dimension of AO-based two-electron integral tensor.

\begin{tabular}{lclc}
\hline \hline Basis set & $\begin{array}{c}\text { \# of basis } \\
\text { functions }\end{array}$ & Block size & $d$ \\
\hline $6-31 \mathrm{G}$ & 18 & $16(11), 40(35), 40(35), 75(71)$ & $171(152)$ \\
$6-31 \mathrm{G}(\mathrm{d}, \mathrm{p})$ & 28 & $4(4), 60(39), 96(75), 96(75), 150(126)$ & $406(319)$ \\
$6-311 \mathrm{G}$ & 26 & $36(22), 84(59), 84(59), 147(116)$ & $351(256)$ \\
$6-311 \mathrm{G}(\mathrm{d}, \mathrm{p})$ & 36 & $4(4), 96(57), 160(102), 160(102), 246(174)$ & $666(439)$ \\
cc-pVDZ & 28 & $4(4), 60(39), 96(76), 96(76), 150(128)$ & $406(323)$ \\
aug-cc-pVDZ & 46 & $16(11), 172(71), 260(134), 260(134), 373(204)$ & $1081(554)$ \\
cc-pVTZ & 60 & $4(4), 348(140), 448(206), 448(206), 582(301)$ & $1830(857)$ \\
aug-cc-pVTZ & 92 & $16(11), 848(201), 1056(299), 1056(299), 1302(412)$ & $4278(1222)$ \\
\hline \hline
\end{tabular}

the decomposition is operated in the MO space. In this sense, the intermediate $I^{\prime}$ will become a permutation matrix, and the cost of the Eq. (17) will be just $\mathcal{O}\left(N_{o}^{2} d_{k}\right)$.

Alternatively, the Eq. (12) can be rewritten in terms of the Coulomb-type integral tensor,

$$
\sum_{e, f}^{N_{v}} \tilde{K}_{a b e f} t_{i j}^{e f}=\sum_{e, f} \tilde{J}_{a e b f} t_{i j}^{e f}=\sum_{k}^{n} \sum_{m}^{N_{k}}\left(Y_{k}(a, e, m) Y_{k}(b, f, m)\right) t_{i j}^{e f},
$$

where

$$
\begin{aligned}
& Y_{k}(a, b, m)=\sum_{x}^{d_{k}} I^{\prime \prime \prime}(a, b, x) L_{J, k}(x, m), \\
& I^{\prime \prime \prime}(a, b, x)=\sum_{\mu, \nu}^{N} C_{\mu, a} C_{\nu, b} P_{J}(\mathcal{P}, x) .
\end{aligned}
$$

135 Again, if the Cholesky decomposition is done on $(a e \mid b f)$, then the intermediate $\mathbf{I}^{\prime \prime \prime}$ will be just a permutation, and the cost of Eq. (19) will be just $\mathcal{O}\left(d_{k} N_{k}\right)$.

Some simple tests have been done in our pilot implementation. Table 1 gives the block information of a permuted AO-based integral tensor, as well 


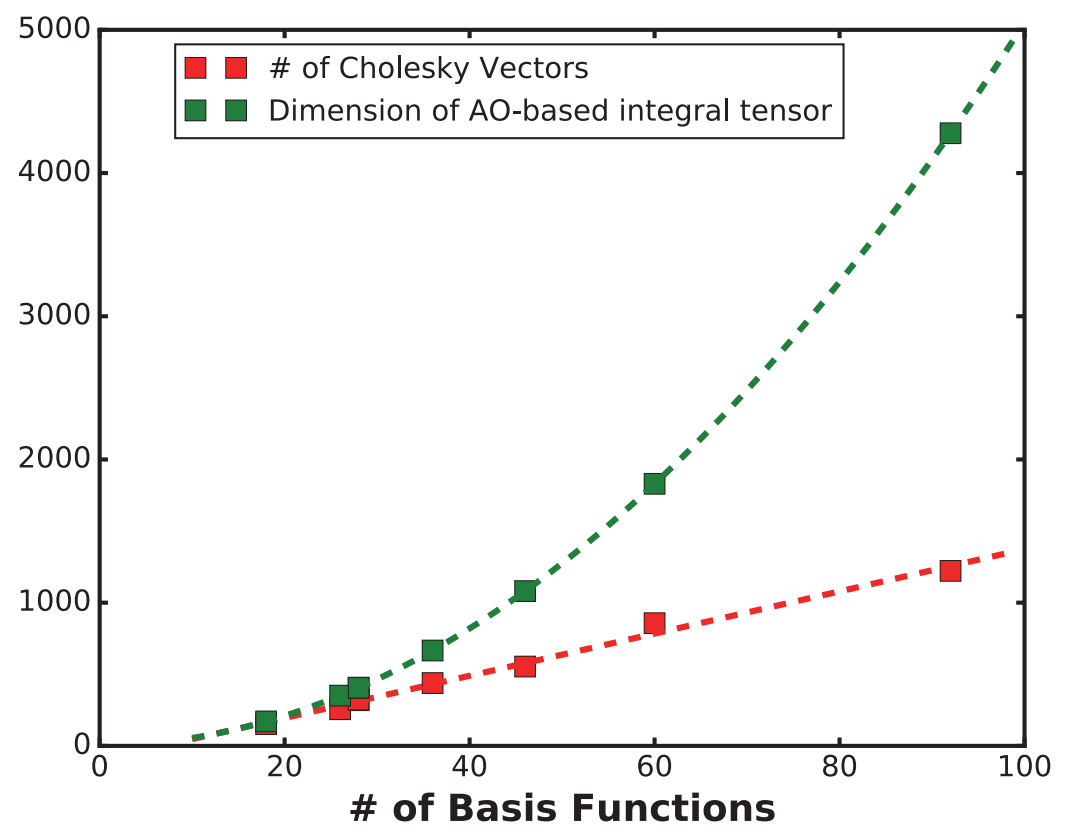

Figure 3: number of the Cholesky vectors and dimension of the integral tensor in AO space increase as the number of the basis functions increases for an $\mathrm{N}_{2}$ molecule. Since the AObased integral tensor $(\mu \nu \mid \lambda \sigma)$ is usually mapped onto a 2 D tensor $(\mathcal{P} \mid \mathcal{Q})$ with $\mathcal{P} \equiv(\mu, \nu)=$ $\mu(\mu-1) / 2+\nu$ and $\mathcal{Q} \equiv(\lambda, \sigma)=\lambda(\lambda-1) / 2+\sigma$, the dimension of the AO-based integral tensor is then becomes $N(N+1) / 2$ with $N$ the number of the basis functions.

as rank information of the generated Cholesky vectors, of $\mathrm{N}_{2}$ molecule with various basis sets. It seems the number of the diagonal blocks in the permuted AO-based integral tensor (four or five blocks for $\mathrm{N}_{2}$ molecule) is not sensitive to the size of the basis sets adapted here. Also, the number of the Cholesky vectors shows an almost linear dependence on the number of the basis functions. For $\mathrm{N}_{2}$ molecule, given a truncation threshold (e.g. $\left.1.0 \times 10^{-10}\right)$, the number of the Cholesky vectors is approximately 14 times larger than the number of the basis functions (see Fig. 3). Thus, one can easily estimate the number of the Cholesky vectors given the same truncation threshold, and compare it with the dimension of the tensor before performing the Cholesky decompositions. 
Table 2 shows the block information of the permuted AO/MO-based inte-

It's worth mentioning that in our pilot implementation we also explored the decomposition of other MO-based two-electron integral tensors, such as $(i j \mid a b)$ and $(i j \mid k l)$. Also, for large subblocks, the size of some Cholesky vectors might 
Table 2: The rank of each block in the block-diagonal AO- and MO-based integral tensors, total number of the Cholesky vectors, $\Delta \mathrm{E}_{\mathrm{MP} 2}$, and $\Delta \mathrm{E}_{\mathrm{L}-\mathrm{CCD}}$ for $\mathrm{N}_{2}$ molecule with cc-pVDZ basis set and various truncation thresholds of incomplete pivoted $\mathrm{CD} . \Delta \mathrm{E}_{\mathrm{MP} 2}$ and $\Delta \mathrm{E}_{\mathrm{L}-\mathrm{CCD}}$ obtained from standard procedure are -0.308904138 a.u. and -0.318634698 a.u., respectively. The deviations are given in parenthesis. The numbers of the basis functions, the occupied orbitals, and the virtual orbitals are 28,7 , and 21 , respectively. The parameter $d$ defines the dimension of two-electron integral tensor.

\begin{tabular}{|c|c|c|c|c|c|c|}
\hline Threshold & Integral type & $d$ & Rank of each block & $\begin{array}{c}\text { \# of Cholesky } \\
\text { vectors }\end{array}$ & $\Delta \mathrm{E}_{\mathrm{MP} 2} /$ a.u. ${ }^{\mathrm{a}}$ & $\Delta \mathrm{E}_{\mathrm{L}-\mathrm{CCD}} / \mathrm{a} \cdot \mathrm{u}$ \\
\hline \multirow[t]{4}{*}{$1.00 \times 10^{-4}$} & $\mathrm{AO},(\mu \nu \mid \lambda \sigma)$ & 406 & $4,17,33,33,55$ & 142 & & \\
\hline & MO, $(i a \mid j b)$ & 147 & $10,19,19,34$ & 82 & -0.308888519 & -0.318623015 \\
\hline & $\mathrm{MO},(a b \mid c d)$ & 231 & $2,3,31,8,23,31,10,31$ & 139 & $\left(1.56 \times 10^{-5}\right)$ & $\left(1.17 \times 10^{-5}\right)$ \\
\hline & $\mathrm{MO},\langle a b \mid c d\rangle$ & 441 & $110,110,86,135$ & 441 & & \\
\hline \multirow[t]{4}{*}{$1.00 \times 10^{-6}$} & $\mathrm{AO},(\mu \nu \mid \lambda \sigma)$ & 406 & $4,27,48,48,84$ & 211 & & \\
\hline & $\mathrm{MO},(i a \mid j b)$ & 147 & $14,29,29,49$ & 121 & -0.308904034 & -0.318634805 \\
\hline & $\mathrm{MO},(a b \mid c d)$ & 231 & $2,3,48,12,32,48,14,42$ & 201 & $\left(1.04 \times 10^{-7}\right)$ & $\left(1.07 \times 10^{-7}\right)$ \\
\hline & $\mathrm{MO},\langle a b \mid c d\rangle$ & 441 & $110,42,110,44,135$ & 441 & & \\
\hline \multirow[t]{4}{*}{$1.00 \times 10^{-8}$} & $\mathrm{AO},(\mu \nu \mid \lambda \sigma)$ & 406 & $4,33,68,68,108$ & 286 & & \\
\hline & $\mathrm{MO},(i a \mid j b)$ & 147 & $14,29,29,49$ & 121 & -0.308904114 & -0.318634688 \\
\hline & $\mathrm{MO},(a b \mid c d)$ & 231 & $2,3,29,27,13,33,27,29,17,46$ & 226 & $\left(2.41 \times 10^{-8}\right)$ & $\left(9.28 \times 10^{-9}\right)$ \\
\hline & $\mathrm{MO},\langle a b \mid c d\rangle$ & 441 & $56,54,42,66,54,56,44,69$ & 441 & & \\
\hline \multirow[t]{4}{*}{$1.00 \times 10^{-10}$} & $\mathrm{AO},(\mu \nu \mid \lambda \sigma)$ & 406 & $4,39,76,76,128$ & 323 & & \\
\hline & MO, $(i a \mid j b)$ & 147 & $16,37,37,56$ & 146 & -0.308904138 & -0.318634707 \\
\hline & $\mathrm{MO},(a b \mid c d)$ & 231 & $2,3,29,28,14,34,28,29,17,46$ & 228 & $\left(<10^{-9}\right)$ & $\left(9.48 \times 10^{-9}\right)$ \\
\hline & $\mathrm{MO},\langle a b \mid c d\rangle$ & 441 & $56,54,42,66,54,56,44,69$ & 441 & & \\
\hline
\end{tabular}

${ }^{a}$ For closed shell systems, $\Delta \mathrm{E}_{\mathrm{MP} 2}=\sum_{i, j}^{N_{o}} \sum_{a, b}^{N_{v}}[2(i a \mid j b)-(i b \mid j a)] t_{i j}^{a b}$, with $t_{i j}^{a b}=\frac{(i a \mid j b)}{\epsilon_{i}+\epsilon_{j}-\epsilon_{a}-\epsilon_{b}}$.

${ }^{\mathrm{b}}$ For closed shell systems, $\Delta \mathrm{E}_{\mathrm{L}-\mathrm{CCD}}=\sum_{i, j}^{N_{o}} \sum_{a, b}^{N_{v}}[2(i a \mid j b)-(i b \mid j a)] t_{i j}^{a b}$, and $t_{i j}^{a b}$ is the solution of the linear double amplitude equation, $\left(\epsilon_{i}+\epsilon_{j}-\epsilon_{a}-\epsilon_{b}\right) t_{i j}^{a b}=(i a \mid j b)+P_{i a}^{j b}\left\{\frac{1}{2} \sum_{c, d}^{N_{v}}\langle a b \mid c d\rangle t_{i j}^{c d}+\frac{1}{2} \sum_{k, l}^{N_{o}}(i k \mid j l) t_{k l}^{a b}-\sum_{k}^{N_{o}} \sum_{c}^{N_{v}}\left[(i k \mid c b) t_{k j}^{a c}+\right.\right.$ $\left.\left.(i k \mid a c) t_{k j}^{c b}-\left(2 t_{k i}^{c a}-t_{i k}^{c a}\right)(k c \mid j b)\right]\right\}$, with $P_{i a}^{j b}(i a \mid j b)=(i a \mid j b)+(j b \mid i a)$. More details can be found in Ref. [35]. 

block-diagonal form, such that the conventional pivoted CD can be performed by decomposing each diagonal blocks. Since the rank of each diagonal block 
Table 3: The MP2 and L-CCD correlation energies of several molecules computed in this work (with double- $\zeta$ basis sets) and corresponding deviations with respect to the results obtained from the standard L-CCD equations. The truncation threshold in incomplete pivoted Cholesky decomposition is set to $1.0 \times 10^{-6}$.

\begin{tabular}{|c|c|c|c|c|c|c|}
\hline & Basis function & $\begin{array}{l}\text { \# of basis } \\
\text { functions }\end{array}$ & $\Delta \mathrm{E}_{\mathrm{MP} 2} /$ a.u. & Dev./a.u. & $\Delta \mathrm{E}_{\mathrm{L}-\mathrm{CCD}} /$ a.u. & Dev./a.u. \\
\hline \multirow[t]{2}{*}{$\mathrm{H}_{2} \mathrm{O}$} & $6-31 \mathrm{G}$ & 13 & -0.128755304 & $3.37 \times 10^{-8}$ & -0.134751072 & $1.48 \times 10^{-8}$ \\
\hline & cc-pVDZ & 24 & -0.203714816 & $5.12 \times 10^{-8}$ & -0.215385757 & $1.62 \times 10^{-\varepsilon}$ \\
\hline \multirow[t]{2}{*}{$\mathrm{HF}$} & $6-31 \mathrm{G}$ & 44 & -0.460652924 & $1.68 \times 10^{-6}$ & -0.477758718 & $1.08 \times 10^{-6}$ \\
\hline & cc-pVDZ & 76 & -0.833712149 & $1.27 \times 10^{-6}$ & -0.861829495 & $4.62 \times 10^{-7}$ \\
\hline \multirow[t]{2}{*}{$\mathrm{C}_{2} \mathrm{H}_{4}$} & 6-31G & 26 & -0.182596654 & $4.26 \times 10^{-7}$ & -0.213306232 & $3.37 \times 10^{-7}$ \\
\hline & cc-pVDZ & 48 & -0.280481739 & $6.58 \times 10^{-7}$ & -0.318648882 & $2.09 \times 10^{-7}$ \\
\hline \multirow[t]{2}{*}{$\mathrm{C}_{4} \mathrm{H}_{6}$} & 6-31G & 48 & -0.359121481 & $1.22 \times 10^{-6}$ & -0.412592537 & $1.38 \times 10^{-7}$ \\
\hline & cc-pVDZ & 86 & -0.545330362 & $1.10 \times 10^{-6}$ & -0.609461647 & $7.32 \times 10^{-8}$ \\
\hline \multirow[t]{2}{*}{$\mathrm{C}_{6} \mathrm{H}_{8}$} & $6-31 \mathrm{G}$ & 70 & -0.536946766 & $1.90 \times 10^{-6}$ & -0.612665634 & $1.04 \times 10^{-6}$ \\
\hline & cc-pVDZ & 124 & -0.811140102 & $1.58 \times 10^{-6}$ & -0.900665100 & $2.54 \times 10^{-7}$ \\
\hline \multirow[t]{2}{*}{ Ethanol } & $6-31 \mathrm{G}$ & 39 & -0.306827760 & $1.02 \times 10^{-6}$ & -0.339692233 & $9.50 \times 10^{-\varepsilon}$ \\
\hline & cc-pVDZ & 72 & -0.486488340 & $6.74 \times 10^{-7}$ & -0.532882784 & $8.60 \times 10^{-8}$ \\
\hline \multirow[t]{2}{*}{ Benzene } & 6-31G & 66 & -0.525618058 & $3.86 \times 10^{-6}$ & -0.581785756 & $3.64 \times 10^{-7}$ \\
\hline & cc-pVDZ & 114 & -0.800034980 & $1.49 \times 10^{-6}$ & -0.864997423 & $4.46 \times 10^{-7}$ \\
\hline \multirow[t]{2}{*}{ Glycine } & $6-31 \mathrm{G}$ & 55 & -0.544052821 & $1.90 \times 10^{-6}$ & -0.564824561 & $5.51 \times 10^{-7}$ \\
\hline & cc-pVDZ & 95 & -0.821994562 & $7.45 \times 10^{-7}$ & -0.863141832 & $4.22 \times 10^{-7}$ \\
\hline \multirow[t]{2}{*}{ Alanine } & 6-31G & 68 & -0.644557271 & $3.92 \times 10^{-6}$ & -0.678839357 & $2.00 \times 10^{-6}$ \\
\hline & cc-pVDZ & 119 & -0.974953969 & $9.87 \times 10^{-7}$ & -1.032578081 & $4.24 \times 10^{-7}$ \\
\hline
\end{tabular}


is smaller than the rank of the original tensor, this can significantly accelerate

the CD procedure. More importantly, the block-diagonal form of the integral tensor can further facilitate the low-rank factorization of the tensor AO-to-MO transformation, as well as the tensor contractions. Our first practice includes the MP2 and L-CCD models. Preliminary tests have shown that even for small molecules block-diagonal form of two-electron integral tensor can be obtained in either the AO or the MO space. The Cholesky vectors of the block-diagonal form in the MO space will have lower rank than those in the AO space. The MP2 and L-CCD energies of some small molecules obtained with this procedure show a very good agreement with the canonical results. The more efficient parallel implementation of this technique and its extension to higher order CC models are under intensive development.

\section{Acknowledgements}

This work has been performed using the Molecular Science Computing Facility (MSCF) in the Environmental Molecular Sciences Laboratory (EMSL) at the Pacific Northwest National Laboratory (PNNL). EMSL is funded by the

${ }_{225}$ Office of Biological and Environmental Research in the U.S. Department of Energy. PNNL is operated for the U.S. Department of Energy by the Battelle Memorial Institute under Contract DE-AC06-76RLO-1830. B. P. acknowledges the Linus Pauling Postdoctoral Fellowship from PNNL.

\section{References}

${ }_{230}^{23}$ [1] F. Coester, Bound states of a many-particle system, Nucl. Phys. 7 (1958) 421-424. doi:http://dx.doi.org/10.1016/0029-5582(58)90280-3. URL http://ww.sciencedirect.com/science/article/pii/ 0029558258902803

[2] F. Coester, H. Kümmel, Short-range correlations in nuclear wave functions, Nucl. Phys. 17 (1960) 477-485. doi:http://dx.doi.org/10.1016/ 
0029-5582(60) 90140-1.

URL http://www.sciencedirect.com/science/article/pii/ 0029558260901401

[3] J. Čížek, On the correlation problem in atomic and molecular systems. calculation of wavefunction components in ursell-type expansion using quantum-field theoretical methods, J. Chem. Phys. 45 (11) (1966) 42564266. doi:http://dx.doi.org/10.1063/1.1727484.

URL http://scitation.aip.org/content/aip/journal/jcp/45/11/ $10.1063 / 1.1727484$

[4] J. Paldus, J. Č́žzek, I. Shavitt, Correlation problems in atomic and molecular systems. iv. extended coupled-pair many-electron theory and its application to the $\mathrm{bh}_{3}$ molecule, Phys. Rev. A 5 (1972) 50-67. doi:10.1103/ PhysRevA.5.50.

URL http://link.aps.org/doi/10.1103/PhysRevA.5.50

[5] G. Purvis, R. Bartlett, A full coupled-cluster singles and doubles model: The inclusion of disconnected triples, J. Chem. Phys. 76 (4) (1982) 19101918. doi:http://dx.doi.org/10.1063/1.443164.

URL http://scitation.aip.org/content/aip/journal/jcp/76/4/10. $1063 / 1.443164$

[6] J. Paldus, X. Li, A Critical Assessment of Coupled Cluster Method in Quantum Chemistry, John Wiley \& Sons, Inc., 2007, pp. 1-175. doi: 10.1002/9780470141694.ch1.

URL http://dx.doi.org/10.1002/9780470141694.ch1

[7] R. J. Bartlett, M. Musiał, Coupled-cluster theory in quantum chemistry, Rev. Mod. Phys. 79 (2007) 291-352. doi:10.1103/RevModPhys.79.291. URL http://link.aps.org/doi/10.1103/RevModPhys.79.291

[8] K. Raghavachari, G. W. Trucks, J. A. Pople, M. Head-Gordon, A fifth-order perturbation comparison of electron correlation theories, Chem. Phys. Lett. 157 (6) (1989) 479 - 483. doi:http: 
//dx.doi.org/10.1016/S0009-2614(89)87395-6.

URL http://www.sciencedirect.com/science/article/pii/ S0009261489873956

[9] F. Weigend, A fully direct ri-hf algorithm: Implementation, optimised auxiliary basis sets, demonstration of accuracy and efficiency, Phys. Chem. Chem. Phys. 4 (2002) 4285-4291. doi:10.1039/B204199P.

URL http://dx.doi.org/10.1039/B204199P

[10] O. Vahtras, J. Almlöf, M. Feyereisen, Integral approximations for lcao-scf calculations, Chem. Phys. Lett. 213 (5) (1993) 514-518. doi:http://dx.doi .org/10.1016/0009-2614(93)89151-7.

URL http://www.sciencedirect.com/science/article/pii/ 0009261493891517

[11] M. Feyereisen, G. Fitzgerald, A. Komornicki, Use of approximate integrals in ab initio theory. an application in mp2 energy calculations, Chem. Phys. Lett. 208 (5) (1993) 359-363. doi :http://dx.doi.org/10.1016/0009-2614(93)87156-W.

URL http://www.sciencedirect.com/science/article/pii/ 000926149387156W

[12] H.-J. Werner, F. R. Manby, P. J. Knowles, Fast linear scaling second-order möller-plesset perturbation theory (mp2) using local and density fitting approximations, J. Chem. Phys. 118 (18) (2003) 8149-8160. doi:http: //dx.doi.org/10.1063/1.1564816.

URL http://scitation.aip.org/content/aip/journal/jcp/118/18/ $10.1063 / 1.1564816$

[13] A. Sodt, J. E. Subotnik, M. Head-Gordon, Linear scaling density fitting, J. Chem. Phys. 125 (19) (2006) 194109. doi:http://dx.doi.org/10.1063/ 1.2370949 .

URL http://scitation.aip.org/content/aip/journal/jcp/125/19/ $10.1063 / 1.2370949$ 
[14] A. P. Rendell, T. J. Lee, Coupled-cluster theory employing approximate integrals: An approach to avoid the input/output and storage bottlenecks, J. Chem. Phys. 101 (1) (1994) 400-408. doi:http://dx.doi.org/10.1063/1.468148.

URL http://scitation.aip.org/content/aip/journal/jcp/101/1/ $10.1063 / 1.468148$

[15] J. L. Whitten, Coulombic potential energy integrals and approximations, J. Chem. Phys. 58 (10) (1973) 4496-4501. doi:http: //dx.doi.org/10.1063/1.1679012.

URL http://scitation.aip.org/content/aip/journal/jcp/58/10/ $10.1063 / 1.1679012$

[16] B. I. Dunlap, J. W. D. Connolly, J. R. Sabin, On some approximations in applications of $x \alpha$ theory, J. Chem. Phys. 71 (8) (1979) 3396-3402. doi:http://dx.doi.org/10.1063/1.438728.

URL http://scitation.aip.org/content/aip/journal/jcp/71/8/10. $1063 / 1.438728$

[17] I. Roeggen, E. Wisloff-Nilssen, On the beebe-linderberg two-electron integral approximation, Chem. Phys. Lett. 132 (2) (1986) 154-160. doi:http://dx.doi .org/10.1016/0009-2614(86)80099-9.

URL http://www.sciencedirect.com/science/article/pii/ 0009261486800999

[18] H. Koch, A. Snchez de Mers, T. B. Pedersen, Reduced scaling in electronic structure calculations using cholesky decompositions, J. Chem. Phys. 118 (21) (2003) 9481-9484. doi:http://dx.doi.org/10.1063/1. 1578621.

URL http://scitation.aip.org/content/aip/journal/jcp/118/21/ $10.1063 / 1.1578621$

[19] N. H. F. Beebe, J. Linderberg, Simplifications in the generation and transformation of two-electron integrals in molecular calculations, Int. J. Quan- 
tum Chem. 12 (4) (1977) 683-705. doi:10.1002/qua.560120408.

URL http://dx.doi.org/10.1002/qua.560120408

325 [20] F. Aquilante, T. B. Pedersen, R. Lindh, Low-cost evaluation of the exchange fock matrix from cholesky and density fitting representations of the electron repulsion integrals, J. Chem. Phys. 126 (19) (2007) 194106. doi:http://dx.doi.org/10.1063/1.2736701.

URL http://scitation.aip.org/content/aip/journal/jcp/126/19/

[21] I. S. Duff, J. K. Reid, J. A. Scott, The use of profile reduction algorithms with a frontal code, Int. J. Numer. Meth. Eng. 28 (11) (1989) 2555-2568. doi:10.1002/nme.1620281106.

URL http://dx.doi.org/10.1002/nme.1620281106

[22] A. George, J. Liu, Computer solution of large sparse positive definite systems, Prentice-Hall series in computational mathematics, Prentice-Hall, 1981.

URL https://books . google.com/books?id=pIYpAQAAMAAJ

[23] C. tools, http://www.culatools.com/blog/2011/12/19/2957/.

[24] K. I. Karantasis, A. Lenharth, D. Nguyen, M. J. Garzarán, K. Pingali, Parallelization of reordering algorithms for bandwidth and wavefront reduction, in: Proceedings of the International Conference for High Performance Computing, Networking, Storage and Analysis, SC '14, IEEE Press, Piscataway, NJ, USA, 2014, pp. 921-932. doi:10.1109/SC.2014.80.

[25] E. Cuthill, J. McKee, Reducing the bandwidth of sparse symmetric matrices, in: Proceedings of the 1969 24th National Conference, ACM '69, ACM, New York, NY, USA, 1969, pp. 157-172. doi:10.1145/800195.805928. URL http://doi.acm.org/10.1145/800195.805928 
[26] G. Karypis, V. Kumar, A parallel algorithm for multilevel graph partitioning and sparse matrix ordering, J. Parallel Distrib. Comput. 48 (1) (1998) 71-95. doi:10.1006/jpdc.1997.1403.

URL http://dx.doi.org/10.1006/jpdc.1997.1403

[27] R. J. Lipton, D. J. Rose, R. E. Tarjan, Generalized nested dissection, SIAM J. Numer. Anal. 16 (2) (1979) 346-358. arXiv:http://dx.doi.org/10. 1137/0716027, doi : 10.1137/0716027.

URL http://dx.doi.org/10.1137/0716027

[28] S. W. Sloan, An algorithm for profile and wavefront reduction of sparse matrices, Int. J. Numer. Meth. Eng. 23 (2) (1986) 239-251. doi:10.1002/ nme. 1620230208 .

URL http://dx.doi.org/10.1002/nme.1620230208

[29] J. Kussmann, C. Ochsenfeld, Linear-scaling method for calculating nuclear magnetic resonance chemical shifts using gauge-including atomic orbitals within hartree-fock and density-functional theory, J. Chem. Phys. 127 (5) (2007) 054103. doi:http://dx.doi.org/10.1063/1.2749509.

URL http://scitation.aip.org/content/aip/journal/jcp/127/5/ $10.1063 / 1.2749509$

[30] S. A. Maurer, L. Clin, C. Ochsenfeld, Cholesky-decomposed density mp2 with density fitting: Accurate mp2 and double-hybrid dft energies for large systems, J. Chem. Phys. 140 (22) (2014) 224112. doi:http://dx.doi. org/10.1063/1.4881144.

URL http://scitation.aip.org/content/aip/journal/jcp/140/22/ $10.1063 / 1.4881144$

[31] G. Moritz, B. A. Hess, M. Reiher, Convergence behavior of the density-matrix renormalization group algorithm for optimized orbital orderings, J. Chem. Phys. 122 (2) (2005) 024107. doi:http://dx.doi.org/10.1063/1.1824891. 
URL http://scitation.aip.org/content/aip/journal/jcp/122/2/ $10.1063 / 1.1824891$

[32] G. K.-L. Chan, M. Head-Gordon, Highly correlated calculations with a polynomial cost algorithm: A study of the density matrix renormalization group, J. Chem. Phys. 116 (11) (2002) 4462-4476. doi:http: //dx.doi.org/10.1063/1.1449459.

URL http://scitation.aip.org/content/aip/journal/jcp/116/11/ 10.1063/1.1449459

[33] W. M. Chan, A. George, A linear time implementation of the reverse cuthill-mckee algorithm, BIT Numer. Math. 20 (1) (1980) 8-14. doi: 10.1007/BF01933580.

URL http://dx.doi.org/10.1007/BF01933580

[34] M. Valiev, E. Bylaska, N. Govind, K. Kowalski, T. Straatsma, H. V. Dam, D. Wang, J. Nieplocha, E. Apra, T. Windus, W. de Jong, Nwchem: A comprehensive and scalable open-source solution for large scale molecular simulations, Comput. Phys. Comm. 181 (9) (2010) 1477-1489. doi:http://dx.doi.org/10.1016/j.cpc.2010.04.018.

URL http://www.sciencedirect.com/science/article/pii/ S0010465510001438

[35] P. Piecuch, S. A. Kucharski, K. Kowalski, M. Musiał, Efficient computer implementation of the renormalized coupledcluster methods: The r-ccsd[t], r-ccsd(t), $\quad \operatorname{cr}-\operatorname{ccsd}[\mathrm{t}], \quad$ and $\quad \mathrm{cr}-$ $\operatorname{ccsd}(\mathrm{t})$ approaches, Comput. Phys. Comm. 149 (2) (2002) 71-96. doi:http://dx.doi .org/10.1016/S0010-4655(02)00598-2.

URL http://www.sciencedirect.com/science/article/pii/ S0010465502005982

[36] V. Khoromskaia, B. N. Khoromskij, R. Schneider, Tensor-structured factorized calculation of two-electron integrals in a general basis, SIAM J. Sci. 
Comput. 35 (2) (2013) A987-A1010. doi:10.1137/120884067.

URL http://dx.doi.org/10.1137/120884067

[37] V. Khoromskaia, B. Khoromskij, Möller-plesset (mp2) energy correction using tensor factorization of the grid-based two-electron integrals, Comp. Phys. Comm. 185 (1) (2014) 2-10. doi:http: //dx.doi.org/10.1016/j.cpc.2013.08.004.

URL http://www.sciencedirect.com/science/article/pii/ S0010465513002658 